\title{
Effectiveness of the 13-valent pneumococcal conjugate vaccine against invasive pneumococcal disease in South African children: a case-control study
}

\author{
Cheryl Cohen, Claire von Mollendorf, Linda de Gouveia, Sarona Lengana, Susan Meiring, Vanessa Quan, Arthermon Nguweneza, David P Moore, \\ Gary Reubenson, Mamokgethi Moshe, Shabir A Madhi, Brian Eley, Ute Hallbaver, Heather Finlayson, Sheeba Varughese, Katherine L O'Brien, \\ Elizabeth R Zell, Keith P Klugman, Cynthia G Whitney, Anne von Gottberg, for the South African IPD Case-Control Study Group*
}

\begin{abstract}
Summary
Background The 13-valent pneumococcal conjugate vaccine (PCV13) was designed to include disease-causing serotypes that are important in low-income and middle-income countries. Vaccine effectiveness estimates are scarce in these settings. South Africa replaced PCV7 with PCV13 in 2011 using a $2+1$ schedule. We aimed to assess the effectiveness of two or more doses of PCV13 against invasive pneumococcal disease in children with HIV infection and in those not infected with HIV.
\end{abstract}

Methods Cases of invasive pneumococcal disease in children aged 5 years or younger were identified through national laboratory-based surveillance. Isolates were serotyped with the Quellung reaction or PCR. We sought in-hospital controls for every case, matched for age, HIV status, and study site. We aimed to enrol four controls for every case not infected with HIV and six controls for every case with HIV infection (case-control sets). With conditional logistic regression, we calculated vaccine effectiveness as a percentage, with the equation 1-[adjusted odds ratio for vaccination] $\times 100$. We included data from an earlier investigation of PCV7 to assess vaccine effectiveness in children exposed to but not infected with HIV and in malnourished children not infected with HIV.

Findings Between January, 2012, and December, 2014, we enrolled children aged 16 weeks or older to our study: 240 were cases not infected with HIV, 75 were cases with HIV infection, 1118 were controls not infected with HIV, and 283 were controls with HIV infection. The effectiveness of two or more doses of PCV13 against PCV13-serotype invasive pneumococcal disease was $85 \%$ (95\% CI 37 to 96 ) among 11 case-control sets of children not infected with HIV and 91\% (-35 to 100) among three case-control sets of children with HIV infection. PCV13 effectiveness among 26 case-control sets of children not infected with HIV was 52\% (95\% CI -12 to 79 ) against all-serotype invasive pneumococcal disease and $94 \%(44$ to 100) for serotype 19A. Vaccine effectiveness against PCV7-serotype invasive pneumococcal disease was $87 \%$ (95\% CI 38 to 97 ) in children exposed to HIV but uninfected and $90 \%$ (53 to 98 ) in malnourished children not infected with HIV.

Interpretation Our results indicate that PCV13 in a $2+1$ schedule is effective for preventing vaccine-type pneumococcal infections in young children not infected with HIV, including those who are malnourished or who have been exposed to HIV. Although the point estimate for PCV13 vaccine effectiveness in children infected with HIV was high, it did not reach significance, possibly because of the small sample size. These findings support recommendations for widespread use of pneumococcal conjugate vaccine in low-income and middle-income countries.

Funding Gavi, The Vaccine Alliance.

Copyright (C) The Author(s). Published by Elsevier Ltd. This is an Open Access article under the CC BY-NC-ND license.

\section{Introduction}

Immunisation with pneumococcal conjugate vaccine is an important strategy to reduce global childhood mortality. Few quantitative results are available for the effect of this vaccine in low-income and middle-income countries because it has not been in use for long and few clinical studies are underway., ${ }^{1,2}$ Policy makers in countries still considering introduction of pneumococcal conjugate vaccine, particularly those in Africa, might be influenced by the effectiveness of the vaccine in routine-use settings. More than $50 \%$ of an estimated 541000 global deaths due to pneumococcus in 2008 occurred in sub-Saharan Africa. HIV infection and in-utero exposure to HIV not resulting in infection are important risk factors for development of pneumococcal disease; approximately $20 \%$ of deaths caused by pneumococcal infection in children younger than 5 years in sub-Saharan Africa were related to HIV in $2000 .^{3}$

The seven-valent pneumococcal conjugate vaccine (PCV7) - the first vaccine of its type to be licensed for routine use- has been replaced globally by higher valency vaccines (PCV10 and PCV13); these newer vaccines were

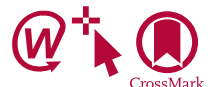

Lancet Glob Health 2017; 5: e359-69

Published Online January 27, 2017 http://dx.doi.org/10.1016/ S2214-109X(17)30043-8

See Comment page e244

*Members listed in appendix Centre for Respiratory Diseases and Meningitis (C Cohen PhD, C von Mollendorf MD L de Gouveia NDMedTech, SLengana MD

A Nguweneza MSc Prof S A Madhi PhD, $A$ von Gottberg PhD), and Division of Public Health Surveillance and Response (S Meiring MD, V Quan MD), National Institute for Communicable Diseases of the National Health Laboratory Service, Johannesburg, South Africa; School of Public Health (C Cohen,

( von Mollendorf), Department of Paediatrics, Chris Hani Baragwanath Academic Hospital (D P Moore MD, Prof S A Madhi), Rahima Moosa Mother and Child Hospital, Department of Paediatrics and Child Health (G Reubenson MD), and Charlotte Maxeke Johannesburg Academic Hospital, Paediatrics Department (S Varughese MD), Faculty of Health Sciences, University of the Witwatersrand, Johannesburg, South Africa; Dr George Mukhari Hospital, Paediatrics Department, Medunsa University, Johannesburg South Africa (M Moshe MD); Department of Science and Technology/National Research Foundation: Vaccine Preventable Diseases, Johannesburg, South Africa (Prof S A Madhi, Prof K P Klugman PhD); Red Cross War Memorial Children's Hospital, and the Department of Paediatrics and Child Health, University of Cape Town, 
Cape Town, South Africa (Prof B Eley MD); Universitas and Pelonomi Hospitals, Department of Paediatrics and Child Health, University of the Free State, Bloemfontein, South Africa (U Hallbauer MD); Tygerberg Hospital, and the Department of Paediatrics and Child Health, Stellenbosch University, Cape Town, South Africa (H Finlayson MD); Johns Hopkins Bloomberg School of Public Health, Johns Hopkins University, Baltimore, MD, USA (Prof K L O'Brien MD); National Center for Immunization and Respiratory Diseases, Centers for Disease Control and Prevention, Atlanta, GA, USA (ER Zell MSc, C G Whitney MD); School of Pathology, University of the Witwatersrand, Johannesberg, South Africa (A von Gottberg); and Hubert Department of Global Health, Rollins School of Public Health, and Division of Infectious Diseases, School of Medicine, Emory University, Atlanta, GA, USA (A von Gottberg) Correspondence to:

Dr Cheryl Cohen, Centre for Respiratory Diseases and Meningitis, National Institute for Communicable Diseases, Sandringham 2131, South Africa cherylc@nicd.ac.za

See Online for appendix

For more on the VIEW-hub platform see http://view-hub.org

\section{Research in context}

\section{Evidence before this study}

We searched PubMed for reports published before April 30, 2016, with the terms "13-valent pneumococcal vaccine" OR "13-valent pneumococcal conjugate vaccine" AND "effectiveness", "efficacy", "impact", OR "invasive pneumococcal disease". We searched for studies that assessed the effectiveness of the 13-valent pneumococcal conjugate vaccine (PCV13) against invasive pneumococcal disease in children younger than 5 years, using a case-control approach. We identified several reports of the effect of the pneumococcal conjugate vaccine but only three in which results were reported of case-control studies of PCV13 effectiveness. Two studies were from the UK, in which the indirect cohort method was used, and one was from the USA, in which a matched case-control approach was used. No studies of the effectiveness of PCV13 were retrieved from low-income or middle-income countries or from countries with high prevalence of HIV, and our search did not identify any studies in which PCV13 effectiveness was assessed in a schedule aligned with WHO's recommended Expanded Programme on Immunisation (EPI). Vaccine effectiveness point estimates against invasive pneumococcal disease caused by serotypes included in the vaccine were $75 \%$ or greater for two or more doses of PCV13 in the UK study and $86 \%$ for one or more dose in the US study. One dose of PCV13 administered in children older than 1 year was also effective in both the UK and US studies. In the report from the UK, greater than $70 \%$ effectiveness of PCV13 was shown against all individual serotypes assessed, except serotypes 3 and 19A. In the US study, $80 \%$ or greater effectiveness of PCV13 was noted against serotypes 3,7F, and 19A.

\section{Added value of this study}

We did a case-control study of PCV13 effectiveness against invasive pneumococcal disease in children not infected with HIV and in those with HIV infection in a middle-income African country. PCV13 was effective against PCV13-serotype invasive pneumococcal disease in children not infected with HIV when implemented in a $2+1$ schedule aligned with WHO's recommended EPI schedule. Furthermore, two or more doses of PCV13 were effective against serotype $19 \mathrm{~A}$, an important cause of replacement pneumococcal disease globally. Although the point estimate for vaccine effectiveness in children infected with HIV was high, it did not reach significance, possibly because of the small sample size.

\section{Implications of all the available evidence}

Our study supports the recommendation for widespread use of pneumococcal conjugate vaccine in low-income and middle-income countries. Combining data from our study with those from a previous study of PCV7 effectiveness showed that two or more doses of PCV7 or PCV13 were effective against PCV7-serotype invasive pneumococcal disease in children exposed to but not infected with HIV and in malnourished children not infected with HIV. These are two of the most important subgroups of children at high risk for serious pneumococcal disease in low-income and middle-income settings with a high prevalence of HIV. These data, when combined with disease rate and vaccine coverage data, might enable estimation of expected vaccine effectiveness in advance of vaccine introduction in other settings. designed to include a larger proportion of serotypes that cause disease in low-income and middle-income settings. Although the vaccine effectiveness of PCV7 has been shown in several settings, ${ }^{4,5}$ and many studies have been published of the effect of PCV7 and PCV13 (as shown on VIEW-hub), the only case-control studies to assess PCV13 effectiveness are from the UK and the USA. ${ }^{6-8}$ Data for effectiveness of PCV13 are needed from middle-income or low-income countries and nations with a high prevalence of HIV. ${ }^{2}$

PCV7 was introduced into the South African routine immunisation programme in April, 2009, using a novel $2+1$ vaccination schedule (first two doses given at age 6 weeks and age 14 weeks, with an additional dose at age 9 months) without catch-up. ${ }^{9}$ PCV13 began to replace PCV7 in June, 2011, administered in the same schedule, and by August, 2011, approximately $80 \%$ of facilities had switched. A limited, single-dose, catch-up campaign among children aged 18 months to 3 years was undertaken in 2011. In 2010, before introduction of PCV13, $82 \%(530 / 650)$ of cases of invasive pneumococcal disease in children younger than 5 years were caused by serotypes in PCV13..$^{10}$ HIV prevalence in South African pregnant women was consistently around 30\% from 2010 to 2012," however, access to interventions for prevention of motherto-child HIV transmission was increased for pregnant women with HIV infection (estimated mother-to-child transmission rate of $2.4 \%$ in 2012) during this period. ${ }^{12}$

We aimed to estimate the effectiveness of two or more doses of PCV13 against invasive pneumococcal disease caused by PCV13 serotypes in children with HIV infections and in those not infected with HIV who were eligible to have received PCV13. In secondary analyses, we aimed to estimate the effectiveness of two or more doses of PCV13 or PCV7 against the seven serotypes in PCV7 in children with HIV infection and in those not infected with HIV, in malnourished children not infected with HIV, and in those exposed to HIV but not infected-groups known to be at high risk for pneumococcal disease.

\section{Methods}

\section{Study population and study design}

We did a matched case-control study at 24 sentinel surveillance hospitals participating in the Group for Enteric, Respiratory and Meningeal Disease Surveillance in South Africa (GERMS-SA) national, laboratory-based, active surveillance programme, ${ }^{13}$ with continuous 
enrolment beginning during the period of PCV7 use (children born from February, 2009, to the end of July, 2011) through to the PCV13 period (children born from August, 2011). Study design and methods of the PCV7 assessment of vaccine effectiveness have been published previously. ${ }^{4}$ For the PCV13 assessment of vaccine effectiveness, we used the same protocol as for the PCV7 analysis but with minor modifications and analysis periods, detailed below.

We defined a case as an episode of illness in an individual with Streptococcus pneumoniae (pneumococcus) from normally sterile-site specimens-eg, cerebrospinal fluid (CSF), blood, pleural fluid, and joint fluid. We judged children eligible for study enrolment if they were: aged 8 weeks or older at specimen collection (cases) or admission (controls); resident in South Africa from age 6 weeks; and in the birth cohort eligible to receive at least one dose of pneumococcal conjugate vaccine through the Expanded Programme on Immunisation (EPI). ${ }^{4}$ Exclusion criteria for cases and controls included absence of verified HIV status, previous enrolment as a case, or enrolment of a twin. We restricted subgroups for PCV13 analysis to infants born from August, 2011 (defined as the PCV13 period), based on calendar time of PCV13 availability.

During the period of use of PCV13, we aimed to enrol four controls for every case not infected with HIV and six controls for every case with HIV infection (casecontrol sets). We matched controls to cases by date of birth (within 1 calendar month for children aged 12 months or younger and within 2 calendar months for children older than 12 months), surveillance site, and HIV status. We judged children eligible for enrolment as a control if they were admitted to or attending the casualty or outpatient department at the same hospital as the case. We excluded children as potential controls if they had a diagnosis of invasive pneumococcal disease, pneumonia, or another non-diarrhoeal vaccine-preventable disease. Every day we compiled lists of potential controls systematically from hospital registers. We attempted to enrol controls as soon as possible after the case-admission date. We only judged hospitalised controls eligible for enrolment if they were identified within $72 \mathrm{~h}$ of their admission. We enrolled controls infected with HIV from neighbouring HIV clinics, selected as clinics that did not actively review vaccination status or offer immunisation with the pneumococcal conjugate vaccine.

We obtained written informed consent from parents or guardians of cases and controls. Institutional review boards at the University of the Witwatersrand, the 24 surveillance sites, the US Centers for Disease Control and Prevention (CDC), and the Johns Hopkins Bloomberg School of Public Health approved the study.

\section{Procedures}

We sent pneumococcal isolates to the National Institute for Communicable Diseases (NICD) in Johannesburg for analysis. We confirmed isolates as pneumococcus with standardised methods. ${ }^{14}$ We used the Quellung reaction to serotype isolates, using specific antisera, including serotypes 6A, 6B, 6C, and 6D (Statens Serum Institut, Copenhagen, Denmark). We confirmed serotypes in samples of CSF from culture-negative but clinically suspicious cases, as well as isolates that lost viability ( $\mathrm{n}=47$ in the PCV13 period), with real-time lytA PCR. ${ }^{14}$ To serotype these samples, we used a PCR serotyping assay consisting of 11 duplex reactions, with an additional primer or probe set for serotype $6 \mathrm{C}$ or $6 \mathrm{D} .{ }^{15}$ We defined PCV7 serotypes $(4,6 \mathrm{~B}, 9 \mathrm{~V}, 14,18 \mathrm{C}, 19 \mathrm{~F}$, and 23F), PCV13 additional serotypes (1, 3, 5, 6A, 7F, and 19A), and non-vaccine serotypes to be mutually exclusive. We defined clinical syndromes hierarchically as meningitis, bacteraemic pneumonia, bacteraemia without focus, and other.

Data collection by interview and record review, and procedures for HIV testing, have been described previously. ${ }^{4}$ We gathered information on exposures such as vaccination status and other potential confounders from 1 month preceding the date of pneumococcal specimen collection (the reference period) from cases and their matched controls. We did HIV testing by ELISA for children aged 18 months or older and by qualitative HIV DNA PCR for children younger than 18 months. We assessed severe immunosuppression based on the percentage of CD4+ cells in the total lymphocyte count (measured by flow cytometry), according to WHO categories. ${ }^{15,16}$ We classified children as being exposed to HIV but not infected if they had documented HIVnegative status but their mother was HIV-positive. We categorised children as malnourished if they had a weight-for-age $Z$ score less than -2 (using 2009 WHO child growth standards, adjusting for prematurity for those born <37 weeks' gestation) or had nutritional oedema. ${ }^{4,17}$ We sought a written immunisation history for all cases and controls from patient-held immunisation records and, if needed, vaccination records at health facilities. If the primary caregiver said the child had never been vaccinated, we recorded the child as unvaccinated.

\section{Statistical analysis}

We assumed vaccine effectiveness against PCV13 serotypes to be $80 \%$ in children not infected with HIV and $65 \%$ in those with HIV infection..$^{4,18}$ For the matched analysis, we assumed a case-control PCV13 vaccination correlation of $0 \cdot 2$. Assuming vaccine coverage of $75 \%$ at a significance level $(\alpha)$ of 0.05 and a power of 0.80 , with a 4:1 match of controls to cases for children not infected with HIV and a 6:1 match for those with HIV infection, we needed to enrol 19 children (cases) with PCV13 serotype disease not infected with HIV and 76 controls, and 40 children with HIV infection and 240 controls.

We present baseline and patients' enrolment data for the PCV13 period (ie, children born from August, 2011, onwards); details for the PCV7 period (ie, children born from February, 2009, to July, 2011) have been 
published previously. ${ }^{4}$ We used data from GERMS-SA to compare the characteristics of enrolled and non-enrolled children (cases) with invasive pneumococcal disease in the PCV13 period. We estimated the matched odds ratio of vaccination (vs no vaccination) in cases and controls, controlling for confounders, using conditional logistic regression. We counted doses of pneumococcal conjugate vaccine only if they were received 14 days or more before the pneumococcal specimen collection date.

Potential confounders that altered the odds ratio of immunisation with pneumococcal conjugate vaccine by more than 10 percentage points were included in multivariable models for analyses. Although no standard cutoff is available for identification of confounders, we opted to use $10 \%$ as a cutoff that would detect meaningful alterations in the odds ratio while maintaining a fairly parsimonious model. We included one set of confounders for children not infected with HIV in the PCV13 period and a second set for those without HIV infection in the combined PCV7 and PCV13 period, to ease comparisons of various estimates for vaccine effectiveness within each group. We did the same for children infected with HIV. We have presented adjustment variables for each analysis as footnotes to the tables. We checked for collinearity and two-way interactions in all final models.

We calculated vaccine effectiveness as a percentage, with the equation 1 -[adjusted matched odds ratio] $\times 100$.
We judged $\mathrm{p}$ values less than 0.05 significant. Analyses were done with Stata statistical software (version 14.1).

For each univariate analysis, we used all available case information. In the multivariable model, we excluded patients with missing data for included variables (data were $>90 \%$ complete for all variables). For the main analyses, the group of children not infected with HIV included all those documented as not infected, including those who were exposed to HIV in utero. We assessed vaccine effectiveness in subgroups for which cases and controls were not matched (eg, HIV exposure, malnutrition) by inclusion of an interaction term in the multivariable model. For the primary objective (effectiveness of two or more doses of PCV13 against invasive pneumococcal disease caused by PCV13 serotypes), we included in the analysis all children aged 16 weeks or older and born in the PCV13 period. For the analysis of vaccine effectiveness in subgroups (eg, malnourished), as well as some analyses of different schedules (appendix p 5), we included children born in both the PCV7 and PCV13 periods to increase statistical power for stratified analyses. For the analysis of effectiveness of one booster dose of PCV13, we included all children eligible to receive PCV13 booster and older than 41 weeks of age.

For analysis of PCV13-specific endpoints, we included individuals born after August, 2011. For secondary

\begin{tabular}{|c|c|c|c|c|c|c|}
\hline & \multicolumn{3}{|c|}{ Not infected with HIV } & \multicolumn{3}{|c|}{ Infected with HIV } \\
\hline & Cases $(n=240)$ & Controls $(n=1118)$ & $\mathrm{p}$ value & Cases $(n=75)$ & Controls ( $n=283$ ) & $\mathrm{p}$ value \\
\hline \multicolumn{7}{|l|}{ Demographics } \\
\hline Age (weeks) & $37(17-106)$ & 36 (17-99) & 0.667 & $48(21-107)$ & $53(20-109)$ & 0.586 \\
\hline Male sex & $144 / 240(60 \%)$ & $668 / 1118(60 \%)$ & 0.943 & $39 / 75(52 \%)$ & $157 / 283(55 \%)$ & 0.591 \\
\hline Female sex & $96 / 240(40 \%)$ & $450 / 1118(40 \%)$ & .. & $36 / 75(48 \%)$ & $126 / 283(45 \%)$ & .. \\
\hline Not black ethnic origin & $35 / 240(15 \%)$ & $185 / 1118(17 \%)$ & 0.454 & $4 / 75(5 \%)$ & $16 / 283(6 \%)$ & 0.914 \\
\hline \multicolumn{7}{|l|}{ Risk factors } \\
\hline Malnutrition* & $84 / 240(35 \%)$ & $341 / 1114(31 \%)$ & 0.184 & $52 / 75(69 \%)$ & $132 / 282(47 \%)$ & 0.001 \\
\hline Low birthweight (<2500 g) & $54 / 233(23 \%)$ & 202/1111 (18\%) & 0.078 & $15 / 72(21 \%)$ & $60 / 280(21 \%)$ & 0.912 \\
\hline Preterm (<37 completed weeks) & $45 / 223(20 \%)$ & $140 / 1054(13 \%)$ & 0.008 & $14 / 67(21 \%)$ & $45 / 262(17 \%)$ & 0.479 \\
\hline Underlying disorders (not HIV) $\dagger$ & $62 / 240(26 \%)$ & $151 / 1118(14 \%)$ & $<0.0001$ & $6 / 75(8 \%)$ & $17 / 283(6 \%)$ & 0.531 \\
\hline Smoking exposure & $51 / 238(21 \%)$ & $192 / 1117(17 \%)$ & 0.122 & $10 / 75(13 \%)$ & 48/283 (17\%) & 0.448 \\
\hline Day care attendance & $36 / 237(15 \%)$ & $161 / 1114(14 \%)$ & 0.770 & $7 / 75(9 \%)$ & $37 / 281(13 \%)$ & $0 \cdot 370$ \\
\hline $\begin{array}{l}\text { Number of children aged }<5 \text { years } \\
\text { in household }\end{array}$ &.. & .. & 0.051 & .. & .. & 0.060 \\
\hline 0 & $130 / 236(55 \%)$ & $688 / 1115(62 \%)$ & .. & $48 / 75(64 \%)$ & $189 / 282(67 \%)$ & .. \\
\hline $1-2$ & $94 / 236(40 \%)$ & $397 / 1115(36 \%)$ &.. & $23 / 75(31 \%)$ & $90 / 282(32 \%)$ & .. \\
\hline$\geq 3$ & $12 / 236(5 \%)$ & $30 / 1115(3 \%)$ &.. & $4 / 75(5 \%)$ & $3 / 282(1 \%)$ & .. \\
\hline $\begin{array}{l}\text { Previous hospital admission } \\
\text { (in past } 12 \text { months) }\end{array}$ & $84 / 341(35 \%)$ & $257 / 1118(23 \%)$ & $<0.0001$ & $43 / 75(57 \%)$ & $114 / 283(40 \%)$ & 0.008 \\
\hline $\begin{array}{l}\text { Upper-respiratory-tract infection in } \\
\text { reference period } \ddagger\end{array}$ & $108 / 236(46 \%)$ & $357 / 1110(32 \%)$ & $<0.0001$ & $50 / 73(68 \%)$ & $87 / 280(31 \%)$ & $<0.0001$ \\
\hline \multirow[t]{2}{*}{ Breastfeds } & $175 / 227(77 \%)$ & 831/1058 (79\%) & 0.630 & $50 / 71(70 \%)$ & $216 / 276(78 \%)$ & 0.164 \\
\hline & & & & & \multicolumn{2}{|c|}{ (Table 1 continues on next pag } \\
\hline
\end{tabular}




\begin{tabular}{|c|c|c|c|c|c|c|}
\hline & \multicolumn{3}{|c|}{ Not infected with HIV } & \multicolumn{3}{|c|}{ Infected with HIV } \\
\hline & Cases $(n=240)$ & Controls $(n=1118)$ & p value & Cases $(n=75)$ & Controls ( $n=283$ ) & p value \\
\hline \multicolumn{7}{|l|}{ (Continued from previous page) } \\
\hline \multicolumn{7}{|l|}{ Socioeconomic factors } \\
\hline Residence in an informal dwelling & $57 / 238(24 \%)$ & $270 / 1118(24 \%)$ & 0.948 & $23 / 75(31 \%)$ & $79 / 283(28 \%)$ & 0.639 \\
\hline Crowding (people per room) &.. & .. & 0.098 & .. &.. & 0.870 \\
\hline$\leq 2$ & $99 / 235(42 \%)$ & $555 / 1115(50 \%)$ & .. & $34 / 75(45 \%)$ & $133 / 282(47 \%)$ & .. \\
\hline $3-4$ & $100 / 235(43 \%)$ & $419 / 1115(38 \%)$ & .. & $30 / 75(40 \%)$ & $114 / 282(40 \%)$ & .. \\
\hline $5-30$ & $36 / 235(15 \%)$ & $141 / 1115(13 \%)$ & .. & $11 / 75(15 \%)$ & $35 / 282(12 \%)$ & .. \\
\hline Maternal education &.. &. & $0 \cdot 163$ &. & .. & 0.709 \\
\hline No secondary & $27 / 237(11 \%)$ & $103 / 1117(9 \%)$ &. & $11 / 73(15 \%)$ & $36 / 282(13 \%)$ & .. \\
\hline Some secondary & $133 / 237(56 \%)$ & $581 / 1117(52 \%)$ &. & $45 / 73(62 \%)$ & $168 / 282(60 \%)$ & .. \\
\hline Completed secondary & $77 / 233(32 \%)$ & $433(39 \%)$ & .. & $17 / 73(23 \%)$ & $78 / 282(28 \%)$ & .. \\
\hline Household has a car & $223 / 1118(20 \%)$ & $39 / 240(16 \%)$ & $0 \cdot 188$ & $13 / 75(17 \%)$ & $30 / 283(11 \%)$ & $0 \cdot 111$ \\
\hline \multicolumn{7}{|l|}{ HIV-related factors } \\
\hline HIV exposed & $53 / 235(23 \%)$ & $316 / 1112(28 \%)$ & 0.067 & NA & NA &.. \\
\hline HIV clinic attendance & NA & NA &. & $14 / 68(21 \%)$ & $201 / 274(73 \%)$ & $<0.0001 \Phi$ \\
\hline HIV stage (WHO classification) & .. &. &. & .. &. & 0.036 \\
\hline 1 & NA & NA &. & $6 / 70(9 \%)$ & $64 / 273(23 \%)$ &. \\
\hline 2 & NA & NA & .. & $2 / 70(3 \%)$ & $11 / 273(4 \%)$ & .. \\
\hline 3 & NA & NA & .. & $33 / 70(47 \%)$ & $114 / 273(42 \%)$ & .. \\
\hline 4 & NA & NA & .. & $29 / 70(41 \%)$ & $84 / 273(31 \%)$ & .. \\
\hline Receiving antiretroviral therapy & NA & NA & .. & $27 / 69(39 \%)$ & $173 / 278(64 \%)$ & $<0.0001$ \\
\hline Severe immunosuppression|| & NA & NA &.. & $29 / 34(85 \%)$ & $99 / 175(57 \%)$ & 0.002 \\
\hline $\begin{array}{l}\text { Receiving trimethoprim- } \\
\text { sulfamethoxazole prophylaxis }\end{array}$ & $3 / 236(1 \%)^{* *}$ & $36 / 1108(3 \%)^{* *}$ & 0.095 & $26 / 74(35 \%)$ & $173 / 283(61 \%)$ & $<0.0001$ \\
\hline Current tuberculosis treatment & $8 / 237(3 \%)$ & $14 / 1112(1 \%)$ & 0.020 & $13 / 75(17 \%)$ & $46 / 282(16 \%)$ & 0.832 \\
\hline \multicolumn{7}{|l|}{ Vaccines received } \\
\hline Hepatitis B at 16 weeks & $181 / 240(75 \%)$ & $952 / 1118(85 \%)$ & $<0.0001$ & $60 / 75(80 \%)$ & $249 / 283(88 \%)$ & 0.074 \\
\hline DTP vaccine at 16 weeks & $157 / 240(65 \%)$ & $863 / 1118(77 \%)$ & $<0.0001$ & $52 / 75(69 \%)$ & $229 / 283(81 \%)$ & 0.030 \\
\hline \multicolumn{7}{|l|}{ PCV13 } \\
\hline No doses & $11 / 240(5 \%)$ & $27 / 1118(2 \%)$ & Reference & $3 / 75(4 \%)$ & $7 / 283(2 \%)$ & Reference \\
\hline One dose & $50 / 240(21 \%)$ & $139 / 1118(12 \%)$ & 0.804 & $14 / 75(19 \%)$ & $24 / 283(8 \%)$ & $0 \cdot 720$ \\
\hline Two doses & $106 / 240(44 \%)$ & $549 / 1118(49 \%)$ & 0.018 & $33 / 75(44 \%)$ & $119 / 283(42 \%)$ & $0 \cdot 583$ \\
\hline Three or more doses & $73 / 240(30 \%)$ & $403 / 1118(36 \%)$ & $<0.0001$ & $25 / 75(33 \%)$ & $133 / 283(47 \%)$ & $0 \cdot 154$ \\
\hline \multicolumn{7}{|l|}{ Age at receipt of PCV13 doses (weeks) } \\
\hline Dose 1 & $6(5-14)$ & $6(5-13)$ & 0.969 & $6(5-26)$ & $6(5-14)$ & 0.079 \\
\hline Dose 2 & $14(13-28)$ & $15(13-26)$ & $0 \cdot 332$ & $16(13-40)$ & $15(13-39)$ & 0.027 \\
\hline Dose 3 & $40(38-46)$ & $39(38-49)$ & 0.539 & $40(32-68)$ & $39(35-54)$ & 0.382 \\
\hline Dose 4 & $44(44-44)$ & $36(36-36)$ & $0 \cdot 317$ & 0 & $42(42-42)$ & .. \\
\hline Influenza vaccine & $3 / 238(1 \%)$ & $5 / 1114(<1 \%)$ & $0 \cdot 138$ & $2 / 74(3 \%)$ & $13 / 281(5 \%)$ & 0.464 \\
\hline \multicolumn{7}{|c|}{  } \\
\hline
\end{tabular}


analyses, in which we assessed vaccine effectiveness against PCV7 serotypes individually or within subgroups (eg, children not infected with HIV and malnourished or exposed to HIV in utero), we included data gathered since the start of the assessment of PCV7 vaccine effectiveness (ie, infants born from February, 2009, onwards).

\section{Role of the funding source}

The funder had no role in study design, data collection, data analysis, data interpretation, or writing of the report. The corresponding author had full access to all data in the study and had final responsibility for the decision to submit for publication.

\section{Results}

Between March, 2010, and December, 2014, we identified 762 eligible children with invasive pneumococcal disease aged 16 weeks or older, of whom 36 were excluded from the analysis (appendix p 3). Of 726 enrolled children (cases), 315 (43\%) were enrolled in the PCV13 period: 240 (76\%) of these were not infected with HIV and 75 (24\%) had HIV infection (table 1). The median age of the 315 enrolled children (cases) eligible to have received two or more PCV13 doses was 39 weeks (IQR 18-107). 183 (58\%) of 315 children were boys and 314 (100\%) were hospitalised. The most common clinical disorders among these children were bacteraemic pneumonia (146/315 [46\%]), meningitis (103/315 [33\%]), and bacteraemia without focus (48/315 $[15 \%])$. Enrolled and non-enrolled cases did not differ by age group, ethnic origin, sex, HIV infection status, specimen type, or province (data not shown).

Among 240 children (cases) not infected with HIV aged 16 weeks or older in the PCV13 period, $24(10 \%)$ had invasive pneumococcal disease caused by PCV7 serotypes and $16(67 \%)$ of these 24 children had received two or more doses of PCV13 (figure, A). In an additional $28(11 \%)$ children, their invasive pneumococcal disease was due to the additional serotypes included in PCV13, and $14(50 \%)$ of these 28 children had received two or more doses of PCV13. 164 (68\%) children had invasive pneumococcal disease caused by non-vaccine serotypes. Among 75 children (cases) with HIV infection aged 16 weeks or older in the PCV13 period, eight $(11 \%)$ had PCV7-type invasive pneumococcal disease and six (75\%) of these eight children had received two or more doses of PCV13 (figure, B). For an additional 13 (17\%) children, their invasive pneumococcal disease was due to the additional serotypes included in PCV13, and seven (54\%) of these 13 children had received two or more doses of PCV13. 48 (64\%) children had invasive pneumococcal disease caused by non-vaccine serotypes.

Between March, 2010, and March, 2015, 5135 eligible age-matched children were identified as potential controls, of whom 1926 were excluded from the analysis (appendix p 4). The remaining 3209 controls were included, of whom 1401 were enrolled in the PCV13 period: 1118 were not infected with HIV and 283 had HIV infection. The median number of controls per case in the PCV13 period was four (IQR 4-5) for children not infected with HIV and three (2-6) for those with HIV infection. The median interval between specimen collection in children (cases) and enrolment of controls was 34 days (IQR 4-201) for controls not infected with HIV and 105 days (12-321) for controls with HIV infection $(\mathrm{p}=0 \cdot 0001)$. Of 1118 controls not infected with HIV aged 16 weeks or older, 417 (37\%) had a diagnosis of diarrhoea, 101 (9\%) had diarrhoea and malnutrition, 104 (9\%) had malnutrition alone, 108 (10\%) had a surgical diagnosis, 69 (6\%) had febrile seizures, and 319 (29\%) had another diagnosis. Of 283 controls with HIV infection aged 16 weeks or older, 203 (72\%) were enrolled during a scheduled HIV clinic visit, 39 (14\%) had malnutrition alone, 18 (6\%) had diarrhoea alone, $16(6 \%)$ had a diagnosis of diarrhoea and malnutrition, and seven $(2 \%)$ had another diagnosis. Controls not infected with HIV and those with HIV infection aged 16 weeks or older and enrolled in the PCV13 period were similar to their respective cases in age, sex, and ethnic origin but differed with respect to other characteristics (table 1). Overall, 1204 (86\%) of 1401 controls and $237(75 \%)$ of 315 cases aged 16 weeks or older had received two or more doses of pneumococcal conjugate vaccine.

Among children not infected with HIV aged 16 weeks or older (ie, after the primary vaccine series at age 6 and 14 weeks) and enrolled in the PCV13 period, the adjusted vaccine effectiveness of two or more doses of PCV13 was $85 \%$ (95\% CI 37 to 96 ) against invasive pneumococcal disease caused by PCV13 serotypes and 92\% (40 to 99) against disease caused only by the six serotypes in PCV13 additional to those in PCV7 (table 2). The adjusted vaccine effectiveness of PCV13 was 52\% (-12 to 79$)$ against all-serotype invasive pneumococcal disease, but this finding was not significant. PCV13 was not effective against non-PCV13 serotype disease $(15 \%,-189$ to 75$)$.

Among children not infected with HIV and enrolled over the whole study period (born from February, 2009, onwards), the adjusted vaccine effectiveness of two or more doses of PCV7 or PCV13 was 78\% (95\% CI 46 to 91 ) against PCV7-serotype invasive pneumococcal disease (table 3). The effectiveness was similar for two doses alone, or two primary doses of PCV7 or PCV13 plus a 9-month dose of PCV7 or PCV13 (appendix p 5) Furthermore, in children who were malnourished, adjusted vaccine effectiveness of this schedule against PCV7-serotype invasive pneumococcal disease was $90 \%$ (95\% CI 53 to 98), and in children exposed in utero to HIV it was $87 \%$ (38 to 97), which is similar to the adjusted vaccine effectiveness noted in children without these conditions (table 3). No protection was noted against PCV7-serotype invasive pneumococcal disease from one dose of PCV7 or PCV13 given at about 6 weeks (vaccine 




Figure: Bar chart showing the serotype of invasive pneumococcal disease and vaccination status of children eligible to receive PCV13 through the routine immunisation programme in South Africa (born after August, 2011)

Bars show the number of children aged 16 weeks or older ( $A$ ) not infected with HIV (total $n=240$ ) and (B) infected with HIV (total $n=75)$, and the number of doses of PCV13 received. *Not subtyped further. †Other non-vaccine serotype confirmed with PCR. ‡Unknown serotypes occurred either because an isolate was not available or because only serogroups could be ascertained with PCR. 


\begin{tabular}{|lccc|}
\hline $\begin{array}{c}\text { Discordant } \\
\text { sets }(\mathbf{n})\end{array}$ & $\begin{array}{l}\text { Unadjusted vaccine } \\
\text { effectiveness (95\% Cl) }\end{array}$ & $\begin{array}{l}\text { Adjusted vaccine } \\
\text { effectiveness (95\% Cl) }\end{array}$ \\
\hline Children aged $\geq 16$ weeks not infected with HIV & & \\
\hline PCV13 serotypes (all) $\dagger$ & 11 & $93 \%(71$ to 98$)$ & $85 \%(37$ to 96$)$ \\
PCV13 serotypes (additional) $\neq$ & 6 & $95 \%(66$ to 69$)$ & $92 \%(40$ to 99$)$ \\
PCV7 serotypes & 5 & $89 \%(11$ to 99$)$ & $74 \%(-183$ to 98$)$ \\
All serotypes & 26 & $67 \%(29$ to 85$)$ & $52 \%(-12$ to 79$)$ \\
Non-PCV13 serotypes & 15 & $30 \%(-114$ to 78$)$ & $15 \%(-189$ to 75$)$ \\
Meningitis PCV13 serotypes & 4 & $94 \%(20$ to 100$)$ & $75 \%(-875$ to 100$)$ \\
Bacteraemic pneumonia PCV13 serotypes & 6 & $89 \%(28$ to 98$)$ & $66 \%(-148$ to 95$)$ \\
Children aged $\geq 16$ weeks with HIV infection & & \\
PCV13 serotypes (all) $\dagger$ & 3 & $94 \%(20$ to 100$)$ & $91 \%(-35$ to 100$)$ \\
PCV13 serotypes (additional) $\neq$ & 3 & $97 \%(55$ to 100$)$ & $82 \%(-155$ to 100$)$ \\
PCV7 serotypes & 0 & NE & NE \\
All serotypes & 9 & $43 \%(-138$ to 87$)$ & $3 \%(-1630$ to 93$)$ \\
Non-PCV13 serotypes & 6 & $-107 \%(-1679$ to 76$)$ & $-558 \%$ (NE to 51$)$ \\
\hline
\end{tabular}

Data are for two or more doses versus no doses. Discordant sets are when at least one control differs from the case with respect to $P C V 13$ vaccination status. $N E=$ not estimable. * For children not infected with HIV, adjustments were for malnutrition, whether the patient had received three doses of diphtheria, tetanus, and pertussis vaccine at 16 weeks of age, and maternal education level. For children with HIV infection, adjustments were for receipt of trimethoprim-sulfamethoxazole prophylaxis, receipt of antiretroviral therapy, and presence of severe immunosuppression on CD4+ T-cell count. †Serotypes in PCV13 were 1, 3, 4, 5, 6A, 6B, 7F, 9V, 14, 18C, 19A, 19F and 23F. $¥$ Additional serotypes in PCV13 but not in PCV7 were 1, 3, 5, 6A, 7F, and 19A.

Table 2: Effectiveness of two or more doses of PCV13 versus no doses against invasive pneumococca disease in children eligible to receive PCV13 through the routine immunisation programme (born after August, 2011), by pneumococcal serotype group and syndrome

\begin{tabular}{|c|c|c|}
\hline & $\begin{array}{l}\text { Unadjusted vaccine effectiveness } \\
(95 \% \mathrm{Cl})\end{array}$ & $\begin{array}{l}\text { Adjusted vaccine effectiveness } \\
(95 \% \mathrm{Cl})^{*}\end{array}$ \\
\hline \multicolumn{3}{|c|}{ Children aged $\geq 16$ weeks not infected with HIV } \\
\hline Overall & $83 \%$ (61 to 92 ) & $78 \%$ (46 to 91 ) \\
\hline Exposed to HIV & $91 \%$ (60 to 98$)$ & $87 \%$ (38 to 97 ) \\
\hline Not exposed to HIV & $81 \%$ (51 to 93 ) & $82 \%$ (44 to 94 ) \\
\hline Malnourished & $85 \%$ (44 to 96$)$ & $90 \%$ (53 to 98$)$ \\
\hline Not malnourished & $81 \%$ (40 to 94 ) & $77 \%$ (17 to 94$)$ \\
\hline \multicolumn{3}{|c|}{ Children aged $\geq 16$ weeks with HIV infection } \\
\hline Overall & $26 \%$ (-98 to 72$)$ & $17 \%(-304$ to 80$)$ \\
\hline Severe immunosuppression $\dagger$ & $-42 \%(-723$ to 76$)$ & $-104 \%(-1433$ to 73$)$ \\
\hline No severe immunosuppression & $75 \%(-31$ to 95$)$ & $66 \%(-94$ to 94$)$ \\
\hline Malnourished & $-40 \%(-390$ to 60$)$ & $-23 \%(-454$ to 73$)$ \\
\hline Not malnourished & $70 \%$ (-140 to 96$)$ & $-7 \%(-3420$ to 97$)$ \\
\hline
\end{tabular}

Vaccine effectiveness is shown for subgroups for which cases and controls were not matched (HIV exposure, malnutrition, severe immunosuppression) and was assessed by inclusion of an interaction term for the subgroup of interest in the multivariable model. For subgroup analyses, 25 discordant sets were identified in individuals not infected with HIV ( 25 included in the analysis of HIV exposure and 24 in the analysis of malnutrition). For subgroup analyses, 18 discordant sets were identified in individuals with HIV infection (14 included in the analysis of severe immunosuppression and 17 in the analysis of malnutrition). *For children not infected with HIV, adjustments were for whether the patient had received three doses of diphtheria, tetanus, and pertussis vaccine at 16 weeks of age and presence of crowding in the home. For children with HIV infection, adjustments were for receipt of antiretroviral therapy and presence of severe immunosuppression on CD4+T-cell count. †Based on CD4+ percentage of total lymphocyte cell count, according to WHO categories. ${ }^{18}$

Table 3: Effectiveness of two or more doses of PCV7 or PCV13 versus no doses against PCV7-serotype invasive pneumococcal disease over the full study period (March, 2010, to December, 2014)

effectiveness $1 \%$, 95\% CI -141 to 59 ), based on information from 34 discordant sets (ie, case-control sets in which at least one control differs from the case with respect to PCV13 vaccination status; appendix $\mathrm{p}$ 5). Too few children older than 9 months received one dose of PCV13 after priming with PCV7, so we could not evaluate this schedule's effectiveness against PCV13 additional serotypes (appendix p 6).

Among children not infected with HIV aged 16 weeks or older and enrolled over the whole study period, who had received either PCV7 or PCV13, adjusted vaccine effectiveness against invasive pneumococcal disease caused by individual serotypes in both PCV7 and PCV13 was $94 \%$ (95\% CI 44 to 100 ) for serotype 14 and $97 \%$ (52 to 100) for serotype 23F (appendix p 7). Vaccine effectiveness for serotypes $6 \mathrm{~B}$ and $19 \mathrm{~F}$ was low, although the numbers of children (cases) contributing to these analyses were small. Restricted to the PCV13 period, the adjusted vaccine effectiveness against additional individual serotypes in PCV13 (not in PCV7) was only significant for serotype 19A (appendix p 8); serotypes 3 , 6A, and 7F could not be assessed.

Among children with HIV infection aged 16 weeks or older and enrolled in the PCV13 period, the adjusted vaccine effectiveness of two or more doses of PCV13 was 91\% (95\% CI -35 to 100) against PCV13-serotype invasive pneumococcal disease and $82 \%$ (-155 to 100) against disease caused only by the six serotypes in PCV13 additional to those in PCV7 (table 2), which was not significant. When including data for doses of PCV7 or PCV13 over the whole study period, adjusted vaccine effectiveness against PCV7-serotype invasive pneumococcal disease overall was lower than in the PCV13 period (17\%, 95\% CI -304 to 80 ) and was not significant (table 3). Moreover, adjusted estimates of vaccine effectiveness were negative for children with HIV infection and severe immunosuppression compared with those without severe immunosuppression and in those with malnutrition, but numbers in each subgroup for these analyses were small and differences were not significant (table 3).

\section{Discussion}

Our analysis shows that two priming doses of PCV13 given in a $2+1$ schedule are effective against PCV13-serotype invasive pneumococcal disease in children not infected with HIV in a low-to-middle income African setting. We also found that the vaccine effectiveness in two important risk groups-children not infected with HIV with malnutrition and children exposed to HIV but not infected-was high and similar to that in children without these conditions. These data provide evidence to support the ongoing introduction and sustained use of pneumococcal conjugate vaccine in low-income and middle-income countries.

PCV13 was licensed on the basis of immunogenicity data; therefore, data for vaccine effectiveness against disease are especially important. Findings of a casecontrol study from the UK, using an indirect cohort approach, showed that two doses of PCV13 in the first 
year of life was $78 \%$ (95\% CI -18 to 95$)$ effective against PCV13-serotype invasive pneumococcal disease. ${ }^{6}$ Moreover, in a case-control study from the USA, one dose or more of PCV13 was $89 \%$ (95\% CI 79 to 94) effective against pneumococcal conjugate vaccineserotype invasive pneumococcal disease. ${ }^{8}$ Similar to these estimates, we found two or more doses of PCV13 to be $85 \%$ (95\% CI 37 to 96 ) effective in children not infected with HIV. Our findings concur with results showing a substantial reduction in incidence of PCV13serotype disease in South African children younger than 5 years after introduction of PCV13 in 2011. ${ }^{5}$

The point estimate for vaccine effectiveness against all-serotype invasive pneumococcal disease in children not infected with HIV was 52\% (95\% CI -12 to 79$)$, similar to the $60 \%$ effectiveness seen in the USA $(95 \% \mathrm{CI}$ $46 \cdot 8-70 \cdot 3)$, but this finding was not significant on adjusted analysis. ${ }^{8}$ In children with HIV infection, the vaccine was also not effective against all-serotype invasive pneumococcal disease serotypes (3\% adjusted, 95\% CI -1630 to 93 ). A major contributing factor to the inability to measure significant effectiveness against all serotypes of invasive pneumococcal disease is probably that this study was done several years after the introduction of pneumococcal conjugate vaccine in South Africa, when vaccine serotypes had already become uncommon because of herd effects, reducing their proportionate contribution to all cases of invasive pneumococcal disease. Additional contributing factors could include possible residual confounding and insufficient power with available case numbers, particularly in the group with HIV infection.

Study findings suggest that vaccine effectiveness might vary according to the serotype included in PCV13.? We documented high PCV13 effectiveness against serotype 19A in children not infected with HIV, an important cause of replacement disease (ie, disease caused by non-vaccine serotypes that become relatively more common after vaccine introduction) in South Africa and globally. ${ }^{5}$ Although not significant, the point estimate of vaccine effectiveness was high for serotype 1 , an important cause of epidemic disease in low-income and middle-income countries and for which efficacy studies have not shown protection conclusively. ${ }^{19}$ The pooled analysis from the PCV7 and PCV13 periods showed high vaccine effectiveness against serotypes 14 and 23F. In studies from other settings, a high vaccine effectiveness has been noted against serotypes 6B and $19 \mathrm{~F}$; we observed low point estimates for vaccine effectiveness but these did not reach significance because of the small sample size. ${ }^{20,21}$ Serotype replacement with non-vaccine serotypes is an important concern. Reassuringly in our study, we did not note negative vaccine effectiveness against non-PCV13 serotypes (adjusted 15\%, 95\% CI -189 to 75).

In children with HIV infection, the high vaccine effectiveness point estimate for two or more doses of
PCV13 against PCV13-serotype invasive pneumococcal disease did not reach significance $(91 \%, 95 \%$ CI -35 to 100). This point estimate result differed from that of our PCV7 vaccine effectiveness assessment in children with HIV infection using the same approach in an earlier period $(-12 \%, 95 \%$ CI -449 to 77$) .{ }^{4}$ Recommendations for an additional 10-week dose of pneumococcal conjugate vaccine for children with HIV infection, made as a result of our earlier analysis, ${ }^{4}$ were never effected because of programmatic challenges of implementing a different vaccine schedule in children infected with HIV and in those uninfected. Differing vaccine effectiveness point estimates could reflect uncertainty because of the low sample size, or improvements in the general health status of infants infected with HIV in South Africa over time, leading to differences in immunological responses to vaccination. Indeed, findings of a subgroup analysis found lower point estimates of effectiveness in severely immunosuppressed and malnourished children with HIV infection, although these were not significant. Importantly, data for the effect of pneumococcal conjugate vaccine show that children with HIV infection had striking reductions in incidence of invasive pneumococcal disease after implementation of pneumococcal conjugate vaccine. ${ }^{5}$

Children exposed to HIV but not infected make up approximately a third of babies born every year in South Africa; they have a high frequency of invasive pneumococcal disease and in-hospital mortality from the disease. ${ }^{22,23}$ PCV13 was effective against PCV7-serotype invasive pneumococcal disease in these children from the PCV7 and PCV13 period, confirming earlier PCV7 findings. ${ }^{4}$ Malnutrition is another common risk condition for invasive pneumococcal disease with severe outcomes in low-income and middle-income countries. ${ }^{24}$ Our study provides the first indication of effectiveness of invasive pneumococcal disease in malnourished children not infected with HIV.

Our study had several limitations. Numbers of cases and controls with HIV infection were low and diminished over the study period because of effective interventions for prevention of mother-to-child HIV transmission. Thus, we had to enrol controls with HIV infection at HIV clinics, potentially biasing estimates of vaccine effectiveness upwards because children at HIV clinics might have better access to vaccination, even though we only included clinics that did not provide immunisation with pneumococcal conjugate vaccine. Controls enrolled at hospitals and clinics rather than in the community could differ in their vaccination status from the general population. Furthermore, the relatively high vaccination coverage in the population meant that few case-control pairs were discordant for vaccination status, even in children not infected with HIV. These low numbers led to wide confidence intervals for some estimates, particularly the analyses in individuals with HIV infection overall and by subgroup. Moreover, among the group not infected with HIV, for less specific endpoints such as all-serotype 
invasive pneumococcal disease and syndrome-specific analyses, confidence intervals were wide after adjustment for potential confounders. These wide confidence intervals limit the precision of results and caution should be used when interpreting the point estimates. Because our study was done at sentinel surveillance sites nationally, it is possible that cases might not be representative of all cases of invasive pneumococcal disease in South Africa. It is also possible that vaccination status was misclassified; however, verified vaccination history for all included cases and controls was obtained and few were excluded based on unavailable vaccine history. The inclusion of children with diarrhoea as controls in a period of rotavirus vaccine availability could potentially have biased estimates towards a lower vaccine effectiveness because controls with rotavirus might have been more likely to be unvaccinated. However, findings of a previous case-control study from South Africa showed that exclusion of patients positive for rotavirus with diarrhoea as potential controls did not change estimates of vaccine effectiveness. ${ }^{25}$ In the adjusted analysis for children not infected with HIV, we included receipt of three doses of diphtheria, tetanus, and pertussis vaccine as a confounder in the multivariable model. Although it is not biologically plausible that this vaccine would directly affect the risk of invasive pneumococcal disease, its inclusion resulted in substantial changes in the vaccine effectiveness (lowering point estimates). The likely explanation is that receipt of the diphtheria, tetanus, and pertussis vaccine is a proxy for unmeasured confounders related to access to health care, which are important to control for. Importantly, we excluded collinearity between receipt of diphtheria, tetanus, and pertussis vaccine and pneumococcal conjugate vaccine.

In conclusion, we show that PCV13 as administered in the South African EPI in a $2+1$ schedule is effective against invasive pneumococcal disease caused by PCV13 serotypes in children not infected with HIV. We also show that pneumococcal conjugate vaccine is effective in children exposed to HIV but not infected and in malnourished children-two important risk groups for pneumococcal disease in low-income and middleincome countries. We were unable to conclusively assess vaccine effectiveness in children with HIV infection; however, data from impact studies of pneumococcal conjugate vaccine show that incidence of invasive pneumococcal disease in children with HIV infection has decreased substantially as a result of the introduction of pneumococcal conjugate vaccine. ${ }^{5}$ The relative contribution of direct and indirect effects to this reduction is unclear. This study provides important data for effectiveness of pneumococcal conjugate vaccine to the South African Department of Health and contributes to our understanding of the effect of PCV13 in routine immunisation programmes in low-income and middleincome countries. ${ }^{4}$ These study results contribute to the evidence base for pneumococcal conjugate vaccine dosing schedules and for programme sustainability.

\section{Contributors}

CC, SAM, KLO'B, ERZ, KPK, CGW, and AvG had the idea for and designed the study. CC, CvM, LdG, SL, SM, VQ, AN, DPM, GR, MM, $\mathrm{BE}, \mathrm{UH}, \mathrm{HF}, \mathrm{SV}$, and AvG contributed to data collection and laboratory processing. All authors contributed to data analysis, data interpretation, and writing or critical review of the report.

\section{Declaration of interests}

$\mathrm{CC}$ has received grant support from Sanofi outside the submitted work. $\mathrm{CvM}$ has received honoraria for speaking from Pfizer outside the submitted work. GR has received local conference support and speaker's fees from Pfizer and Sanofi Aspen outside the submitted work. SAM has received grant support and honoraria for participation in a speaker's bureau and as a scientific adviser to GlaxoSmithKline and Pfizer outside the submitted work. KLO'B has received grant support from Pfizer and GlaxoSmithKline outside the submitted work. UH has received personal fees for a lecture and travel fees from Sanofi, outside the submitted work. AvG has received grant support from Gavi, the Vaccine Alliance, related to the submitted work; grant support from the US Centers for Disease Control and Prevention and Pfizer outside the submitted work; and travel grants from Pfizer, Sanofi, and Novartis outside the submitted work. LdG, SL, SM, VQ, AN, DPM, MM, BE, HF, SV, ERZ, KPK, and CGW declare no competing interests.

\section{Acknowledgments}

We thank the participants and their caregivers; GERMS-SA surveillance officers for enrolling participants and obtaining vaccination histories; laboratory staff throughout South Africa for submitting isolates to the National Institute for Communicable Diseases (NICD); and staff at the Centre for Respiratory Diseases and Meningitis Laboratory at NICD for processing and characterising isolates. Support for this project was provided by Gavi, The Vaccine Alliance through the Program for Appropriate Technology in Health (PATH) and the US Centers for Disease Control and Prevention (CDC), Surveillance for invasive pneumococcal disease in infants (cooperative agreement number 1U19GH000571-04). The views expressed by the authors do not necessarily reflect the views of Gavi, CDC, or PATH.

\section{References}

1 International Vaccine Access Center (IVAC). Vaccine Information Management System (VIMS) report: global vaccine introduction. March, 2014. http://www.jhsph.edu/research/centers-andinstitutes/ivac/view-hub/IVAC-VIMS-Report-2014-Mar.pdf (accessed Dec 8, 2016).

2 Mackenzie GA, Hill PC, Jeffries DJ, et al. Effect of the introduction of pneumococcal conjugate vaccination on invasive pneumococcal disease in The Gambia: a population-based surveillance study. Lancet Infect Dis 2016; 16: 703-11.

3 WHO. Estimated Hib and pneumococcal deaths for children under 5 years of age, 2008. Dec 1, 2013. http://www.who.int/ immunization/monitoring_surveillance/burden/estimates/ Pneumo_hib/en/ (accessed Dec 8, 2016).

4 Cohen C, von Mollendorf C, de Gouveia L, et al. Effectiveness of 7-valent pneumococcal conjugate vaccine against invasive pneumococcal disease in HIV-infected and -uninfected children in South Africa: a matched case-control study. Clin Infect Dis 2014; 59: 808-18.

5 von Gottberg A, de Gouveia L, Tempia S, et al. Effects of vaccination on invasive pneumococcal disease in South Africa. N Engl J Med 2014; 371: 1889-99.

6 Miller E, Andrews NJ, Waight PA, Slack MP, George RC. Effectiveness of the new serotypes in the 13-valent pneumococcal conjugate vaccine. Vaccine 2011; 29: 9127-31.

7 Andrews NJ, Waight PA, Burbidge P, et al. Serotype-specific effectiveness and correlates of protection for the 13-valent pneumococcal conjugate vaccine: a postlicensure indirect cohort study. Lancet Infect Dis 2014; 14: 839-46.

8 Moore MR, Link-Gelles R, Schaffner W, et al. Effectiveness of 13-valent pneumococcal conjugate vaccine for prevention of invasive pneumococcal disease in children in the USA: a matched case-control study. Lancet Respir Med 2016; 4: 399-406.

9 Madhi SA, Cohen C, von Gottberg A. Introduction of pneumococcal conjugate vaccine into the public immunization program in South Africa: translating research into policy. Vaccine 2012; 30 (suppl 3): C21-27. 
10 Respiratory and Meningeal Pathogens Reference Unit, National Institute for Communicable Diseases. GERMS-SA surveillance report: Streptococcus pneumoniae. Communicable Diseases Surveillance Bulletin, vol 9, no 2, pp 50-53. May, 2011. http://www.nicd.ac.za/assets/files/Bulletin\%20May\%202011.pdf (accessed Dec 8, 2016).

11 Department of Health, Republic of South Africa. The 2011 national antenatal sentinel HIV \& syphillis prevalence survey in South Africa. 2012. https://www.health-e.org.za/wp-content/ uploads/2013/05/f0980fb5107a7ce543a8bd5730e52333.pdf (accessed Dec 8, 2016).

12 Sherman GG, Lilian RR, Bhardwaj S, Candy S, Barron P. Laboratory information system data demonstrate successful implementation of the prevention of mother-to-child transmission programme in South Africa. S Afr Med J 2014; 104 (3 suppl 1): 235-38.

13 von Gottberg A, Cohen C, de Gouveia L, et al. Epidemiology of invasive pneumococcal disease in the pre-conjugate vaccine era: South Africa, 2003-2008. Vaccine 2013; 31: 4200-08.

14 Ruoff KL, Whiley RA, Beighton D. Streptococcus. In: Murray PR, Baron EJ, Jorgensen JH, Yolken RH, eds. Manual of Clinical Microbiology. Washington DC: ASM Press, 2006: 405-21.

15 Glencross D, Scott LE, Jani IV, Barnett D, Janossy G. CD45-assisted PanLeucogating for accurate, cost-effective dual-platform CD4+ T-cell enumeration. Cytometry 2002; 50: 69-77.

16 WHO, HIV/AIDS Programme. WHO case definitions of HIV for surveillance and revised clinical staging and immunological classification of HIV-related disease in adults and children. 2007. http://www.who.int/hiv/pub/guidelines/HIVstaging150307.pdf (accessed Dec 8, 2016).

17 WHO. WHO child growth standards and the identification of severe acute malnutrition in infants and children: a joint statement by the World Health Organization and the United Nations Children's Fund. 2009. http://apps.who.int/iris/ bitstream/10665/44129/1/9789241598163_eng.pdf?ua=1 (accessed Dec 8, 2016).
18 Klugman KP, Madhi SA, Huebner RE, Kohberger R, Mbelle N, Pierce N. A trial of a 9-valent pneumococcal conjugate vaccine in children with and those without HIV infection. N Engl J Med 2003; 349: $1341-48$.

19 von Mollendorf C, Cohen C, Tempia S, et al. Epidemiology of serotype 1 invasive pneumococcal disease, South Africa, 2003-2013. Emerg Infect Dis J 2016; 22: 261.

20 Whitney CG, Pilishvili T, Farley MM, et al. Effectiveness of seven-valent pneumococcal conjugate vaccine against invasive pneumococcal disease: a matched case-control study. Lancet 2006; 368: $1495-502$.

21 Andrews N, Waight PA, Borrow R, et al. Using the indirect cohort design to estimate the effectiveness of the seven valent pneumococcal conjugate vaccine in England and Wales. PLoS One 2011; 6: e28435.

22 von Mollendorf C, Cohen C, de Gouveia L, et al, for the South African IPD Case-Control Study Group. Risk factors for invasive pneumococcal disease among children less than 5 years of age in a high HIV-prevalence setting, South Africa, 2010 to 2012. Pediatr Infect Dis J 2015; 34: 27-34.

23 Von Mollendorf C, von Gottberg A, Tempia S, et al, for the Group for Enteric, Respiratory and Meningeal Disease Surveillance in South Africa (GERMS-SA). Increased risk for and mortality from invasive pneumococcal disease in HIV-exposed but uninfected infants aged $<1$ year in South Africa, 2009-2013. Clin Infect Dis 2015; 60: $1346-56$.

24 Ginsburg AS, Izadnegahdar R, Berkley JA, Walson JL, Rollins N, Klugman KP. Undernutrition and pneumonia mortality. Lancet Glob Health 2015; 3: e735-36.

25 Madhi SA, Groom M, Zar HJ, et al. Effectiveness of pneumococcal conjugate vaccine against presumed bacterial pneumonia hospitalisation in HIV-uninfected South African children a case-control study. Thorax 2015; 70: 1149-55. 